\title{
Desenvolvimento Sustentável e Responsabilidade Social Corporativa: uma análise bibliométrica da produção científica internacional
}

\section{Sustainable Development and Corporate Social Responsibility: a bibliometric analysis of International Scientific Production}

\author{
Lisilene Mello da Silveira' \\ Maira Petrini ${ }^{1}$
}

\begin{abstract}
Resumo: Tem-se discutido a Responsabilidade Social Corporativa buscando-se aumentar a compreensão sobre o papel das empresas no contexto do Desenvolvimento Sustentável. Mas quais as problemáticas em evidência atualmente e para onde apontam? Este trabalho analisou as publicações científicas internacionais relacionadas às temáticas Desenvolvimento Sustentável e Responsabilidade Social Corporativa, proporcionando um mapa dos estudos publicados. A partir da análise bibliométrica das pesquisas publicadas na Web of Science entre 1998 e 2015 , foram localizados 197 artigos, escritos por 402 autores, vinculados a 246 instituições de 43 países. Buscando critérios de relevância e atualidade, organizaram-se os artigos em dois grupos: artigos mais citados e artigos mais recentes. Foram analisadas as principais contribuições dessas publicações, identificando-se sete problemáticas principais: Ontologia; Fatores para adoção de iniciativas de sustentabilidade; Avaliação de desempenho; Impactos sociais e/ou ambientais; Relatórios de sustentabilidade; Ensino; e Sustentabilidade como estratégia. Para cada problemática são sugeridas lacunas para futuras pesquisas.
\end{abstract}

Palavras-chave: Desenvolvimento sustentável; Responsabilidade social corporativa; Bibliometria.

\begin{abstract}
Corporate social responsibility has been discussed with the objective of improving the understanding of the performance of organizations in the context of sustainable development. But, what are the highlighted problems in current scientific literature and where are they pointing to? This study analyzed international scientific publications related to Sustainable Development and Corporate Social Responsibility, providing a map of published studies. The bibliometric analysis of studies published in Web of Science from 1998 to 2015 found 197 articles, written by 402 authors, linked to 246 institutions from 43 countries. Looking for relevance criteria and currentness, literature analysis organized the studies into two groups: the most mentioned and the most recent articles. Through the analysis of the main contributions of these publications, it was possible to identify seven key problems: Ontology, Factors for adoption of sustainable initiatives, Performance evaluation, Social and/or environmental impacts, Sustainability reports, Education and Sustainability as a strategy. Opportunities for future research on each problem are suggested.
\end{abstract}

Keywords: Sustainable development; Corporate social responsibility; Bibliometrics.

\section{Introdução}

A preocupação em suprir as necessidades da geração presente sem afetar as gerações futuras no atendimento de suas necessidades configura o conceito Desenvolvimento Sustentável. O papel das organizações como agentes que usufruem dos recursos existentes, sendo assim responsáveis por seu uso de forma sustentável, faz parte dessa preocupação e manifesta-se sob o tema Responsabilidade Social Corporativa.

Desde o início dessa discussão até os dias atuais, é crescente a pluralidade de termos relacionados à temática sustentabilidade (Lyra et al., 2009; Laruccia,
2012; Souza \& Ribeiro, 2013). Quando debruçam-se sobre o tema, pesquisadores e acadêmicos também ampliam a diversidade com novos assuntos e problemáticas. Nesse contexto surge a questão de pesquisa deste artigo: Quais problemáticas estão em evidência na produção científica internacional e para onde apontam? Sendo assim, o objetivo deste artigo foi analisar as publicações científicas internacionais relacionadas às temáticas Desenvolvimento Sustentável e Responsabilidade Social Corporativa, proporcionando um mapa das problemáticas dos estudos publicados nessas áreas.

${ }^{1}$ Faculdade de Administração, Contabilidade e Economia - FACE, Pontifícia Universidade Católica do Rio Grande do Sul - PUCRS, Av. Ipiranga, 6681, Partenon, CEP 90619-900, Porto Alegre, RS, Brasil, e-mail: lisilene.silveira@hotmail.com; maira.petrini@pucrs.br Recebido em Maio 15, 2016 - Aceito em Ago. 29, 2016

Suporte financeiro: Nenhum. 
A escolha das palavras Desenvolvimento Sustentável e Responsabilidade Social Corporativa buscou delimitar a pluralidade de termos, focando na intersecção entre o Desenvolvimento Sustentável e o papel das organizações dentro dele, manifestado na expressão Responsabilidade Social Corporativa. A opção pela análise da produção científica internacional embasou-se no destaque dado às publicações internacionais nas referências dos próprios pesquisadores brasileiros (Souza \& Ribeiro, 2013; Iritani et al., 2015). Além disso, os periódicos internacionais têm mais amplitude de circulação e prestígio, por serem publicados em língua inglesa (Goulart \& Carvalho, 2008). Dessa forma, foi realizada uma análise bibliométrica das pesquisas publicadas na base de dados Web of Science - Social Sciences Citation Index (SSCI), no período de 1945 a 2015.

Os resultados mostraram que essa literatura é relativamente recente, verificando-se a partir de 2009 um contínuo e crescente interesse na elaboração de pesquisas sobre Desenvolvimento Sustentável e Responsabilidade Social Corporativa. Entre outros, foram identificados os periódicos com mais artigos publicados, os periódicos com mais citações e os autores com mais citações. A análise final identifica sete problemáticas em que as publicações analisadas foram classificadas e aponta as principais contribuições de cada uma delas. Por fim, este artigo oferece um panorama das pesquisas em Desenvolvimento Sustentável e Responsabilidade Social Corporativa, apresentando uma taxonomia das problemáticas presentes na literatura corrente e insights para futuras pesquisas.

O artigo se estrutura, além desta introdução, em outras quatro partes. Na seção 2 são apresentados alguns aspectos que descrevem o campo do Desenvolvimento Sustentável e o da Responsabilidade Social Corporativa. Na seção 3 são descritos os procedimentos metodológicos adotados para realização deste trabalho. Nas seções 4 e 5 são apresentados os principais resultados. A seção 6 propõe uma discussão em relação às problemáticas identificadas e, na seção 7, são expostas as considerações finais, seguidas da lista de referências utilizadas neste trabalho.

\section{Desenvolvimento Sustentável e Responsabilidade Social Corporativa}

O conceito Desenvolvimento Sustentável é posto em discussão desde 1960, quando ocorreram a Conferência da Biosfera em Paris e o surgimento da Organização Não Governamental Clube de Roma, em 1968 (Barros, 2007).

Na década de 1980, a ONU retomou o debate das questões ambientais e a primeira-ministra da Noruega, Gro Harlem Brundtland, chefiou a Comissão Mundial sobre o Meio Ambiente e Desenvolvimento, para estudar o assunto. $\mathrm{O}$ documento final denominou-se Nosso Futuro Comum, também conhecido como Relatório Brundtland, o qual propõe o Desenvolvimento Sustentável como aquele cujo objetivo é suprir as necessidades da geração presente sem afetar as gerações futuras no suprimento das delas (Brundtland, 1987).

A preocupação com questões sociais e ambientais, inserida no contexto de Desenvolvimento Sustentável, reflete-se nas organizações através da Responsabilidade Social Corporativa. Segundo Carroll (1979), a responsabilidade social das organizações engloba quatro dimensões: econômicas, legais, éticas e discricionárias, que a sociedade tem das organizações em um determinado ponto no tempo. Elkington (1997), através do conceito do Resultado Tríplice (Triple Bottom-Line), propõe um modelo para as organizações interpretarem a sustentabilidade através da integração de três dimensões: econômica, ambiental e social.

Os autores aqui resgatados podem ser vistos como um pano de fundo para a compreensão dos conceitos Desenvolvimento Sustentável e Responsabilidade Social Corporativa. Entretanto, a literatura evidencia a diversidade de conceitos e, consequentemente, de problemáticas presentes nas publicações mais recentes. Pesquisas sobre a produção científica nacional e internacional, em diferentes temáticas, têm sido conduzidas com o intuito de prover mapas dos conhecimentos desenvolvidos (Bignetti \& Paiva, 2002; Melo \& Andreassi, 2010; Souza \& Ribeiro, 2013).

Mais especificamente nas temáticas desta pesquisa, em uma análise das publicações brasileiras na área de responsabilidade social empresarial nos Encontros da ANPAD de 1997 a 2007, Moretti \& Campanário (2009) apontaram a reprodução das mesmas ideias e pouca maturidade científica dos autores no tema. Em um olhar mais voltado para os padrões de colaboração no campo da sustentabilidade, Yarime et al. (2010) apontam que a mesma é crescente e tende a ser realizada entre países que estão geograficamente mais próximos. Diversas temáticas foram identificadas no mapeamento realizado por Lu \& Liu (2014), o qual visava compreender os papéis fundamentais da difusão do conhecimento sobre Responsabilidade Social Corporativa, tais como: relação entre responsabilidade social e resultado financeiro corporativo, questões sociais envolvidas, como capacidade de resposta social, responsabilidade social abrangendo dimensões econômicas, jurídicas, éticas e discricionárias no desempenho do negócio da empresa. No mapeamento sobre sustentabilidade empresarial, realizado pelos autores Montiel \& Delgado-Ceballos (2014), assuntos como Responsabilidade Social Corporativa, desempenho social corporativo, estratégias ambientais e desempenho ambiental são frequentemente encontrados. Os resultados mostram que sustentabilidade empresarial ainda está 
em desenvolvimento e diversos objetos de estudo podem emergir para aprofundamento.

Essas duas últimas pesquisas convergem com nosso objetivo de compreender quais problemáticas estão em evidência na produção científica internacional. Buscou-se um panorama mais quantitativo, apresentando, por exemplo, os periódicos com mais artigos publicados, os periódicos com mais citações e os autores com mais publicações, bem como um mais qualitativo, analisando-se cada publicação e buscando-se identificar as problemáticas e as contribuições principais. O desenvolvimento do estudo bibliométrico é detalhado a seguir.

\section{Procedimentos metodológicos}

Esta pesquisa caracteriza-se como descritiva, com abordagens qualitativas. As técnicas bibliométricas possibilitam empregar indicadores para estabelecer prognósticos e tendências da produção científica nos diversos campos de pesquisa (Machado, 2007; Lazzarotti et al., 2011), possibilitando realizar análises dos artigos relevantes de áreas e temas de pesquisa (Santos et al., 2011). Tendo por base os trabalhos de Crossan \& Apaydin (2010) e Tranfield et al. (2003), o estudo foi realizado em duas etapas: 1) Busca sistemática e 2) Análise sistemática da literatura, descritos a seguir.

\subsection{Etapa 1: busca sistemática da literatura}

Nesta etapa definiu-se a base de dados, assim como as palavras-chave, os campos de buscas e os filtros de refinamento. A definição de qual literatura será analisada é fundamental, pois define o escopo da pesquisa e impacta na sua validade (Singleton \& Straits, 1999). A escolha da base Web of Science - Social Sciences Citation Index (WoS-SSCI) considerou o fato de esse ser um dos mais abrangentes banco de dados de periódicos revisados por pares representativos e reconhecidos pela comunidade científica internacional, com foco na produção acadêmico-científica das áreas associadas às ciências sociais aplicadas, além de incorporar ferramentas bibliométricas e de análise de citações, alinhadas à utilização de procedimentos bibliométricos (Crossan \& Apaydin, 2010; Brambilla \& Stumpf, 2012; Kurtz et al., 2013; Watanuki et al., 2014). Foi considerada a totalidade dos artigos localizados, acessados a partir do Portal de Periódicos da Capes, compreendendo o período de 1945, primeiro ano disponível na base, até outubro de 2015. Como critérios de busca, os termos utilizados foram Corporate Social Responsibility AND Sustainable Development, buscados em tópicos, os quais permitem localizar os termos nos títulos, palavras-chave e resumo nos artigos.

Com esses procedimentos foram encontradas 360 publicações. Em seguida, para refinamento da busca, foi aplicado o filtro da área de pesquisa, quando se considerou Business e Management e, como tipos de documentos, Articles e Review, o que resultou em 199 artigos. Foram considerados apenas artigos em inglês, finalizando-se a seleção com 197 artigos. Os dados desses foram importados para o software HistCite $^{\circledR}$, o qual auxiliou na análise.

\subsection{Etapa 2: análise sistemática da literatura}

As obras foram organizadas em dois grupos: artigos mais citados e artigos mais recentes, visando a identificação de artigos relevantes e atuais na temática de pesquisa.

No grupo dos artigos mais citados foram selecionados os 13 artigos mais citados, os quais correspondem, sozinhos, a $50 \%$ do total das citações. O grupo dos artigos mais recentes considerou inicialmente todos os artigos publicados no período de 2013 a 2015, critério adotado com base em outros trabalhos: Crossan \& Apaydin (2010) e Kurtz et al. (2013). Por se tratar de publicações recentes, nas quais o número de citações normalmente não é significativo e não serve para selecionar artigos relevantes em uma temática (Crossan \& Apaydin, 2010), o critério adotado para seleção foi filtrar os artigos publicados no período 2013-2015 em periódicos que tiveram no mínimo duas publicações na temática. Vinte periódicos foram considerados, resultando em 75 artigos.

Todos os artigos foram lidos na íntegra e tiveram a abordagem metodológica, a problemática e as principais contribuições analisadas.

\section{Resultados da busca sistemática de literatura}

Foram encontrados 197 artigos sobre Desenvolvimento Sustentável e Responsabilidade Social Corporativa. Essas obras estão publicadas em 51 periódicos e foram escritas por 402 autores vinculados a 246 instituições, localizadas em 43 países distintos. Os artigos citam 10.578 referências e 681 palavras-chave.

A Figura 1 apresenta a distribuição das publicações no tempo. O primeiro artigo publicado acerca do Desenvolvimento Sustentável e Responsabilidade Social Corporativa intitula-se Corporate Environmental Responsibility (Desjardins, 1998). O autor promove um debate de especialistas em ética de negócios e filósofos ambientais, relacionando os assuntos com a responsabilidade social empresarial. $\mathrm{O}$ autor ressalta que a responsabilidade social das empresas deriva das responsabilidades éticas, destacando a fragilidade de se incorporar limites morais nas atividades empresariais, assim como as preocupações ambientais, com base no valor da economia de mercado. O segundo artigo mais antigo foi Sustainable development: The ethics 


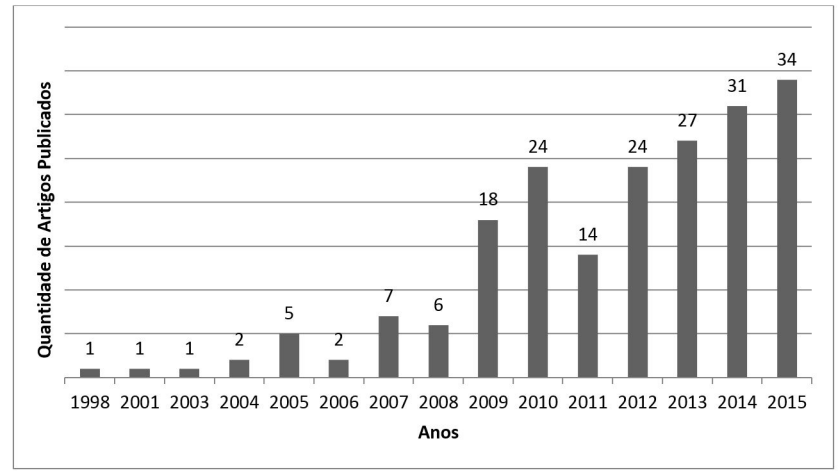

Figura 1. Distribuição, por ano, das publicações sobre desenvolvimento sustentável e RSC (1998-2015). Fonte: Elaboração dos autores, baseada em dados da Web of Science - WoS.

support the economics, de autoria de Payne \& Raiborn (2001). Nesse artigo é destacado que as empresas estão num processo de conscientização dos aspectos ambientais, por meio do posicionamento das empresas na cadeia de valor e também da influência de seus fornecedores e clientes. O comportamento empresarial ao lidar com essas questões é percebido por meio da escolha do processo produtivo, que deve ir além do cumprimento das leis, incorporando a implantação de uma política ambiental própria. Tal política, segundo os autores, reflete-se na cultura corporativa, na qual os gestores estão bem conscientes de que a ética nos negócios é um bom negócio. Para Payne \& Raiborn (2001), isso garante a sobrevivência dessas empresas no longo prazo.

Os elementos discutidos nesses dois artigos mais seminais, como ética, conscientização ambiental, cultura e valor da economia, ainda se mostram presentes nos dias atuais, confirmando que essas questões permanecem como o pano de fundo da discussão de RSC nas organizações.

Publicações nessa área vêm ocorrendo anualmente desde 2003, sendo que os únicos três anos em que não houve publicações, considerando todo o período apresentado, foram 1999, 2000 e 2002. A partir de 2009 percebe-se o crescimento na quantidade de publicações sobre as temáticas, sendo que nos últimos seis anos foi publicado um total de 154 artigos, praticamente $78 \%$ das publicações sobre Desenvolvimento Sustentável e Responsabilidade Social Corporativa.

A Tabela 1 lista os periódicos e a quantidade de publicações e de citações, sendo o critério de corte no mínimo duas publicações ou citações por periódico. O somatório dos artigos dos periódicos com mais publicações resulta 166 artigos, o que corresponde a $84 \%$ da quantidade total de trabalhos encontrados nesta pesquisa. Journal of Business Ethics e Corporate Social Responsibility and Environmental Management, respectivamente com 43 e 39 publicações, são os periódicos que mais publicaram artigos referentes às temáticas Desenvolvimento Sustentável e Responsabilidade Social Corporativa. O periódico Corporate Social Responsibility and Environmental Management apresentou o maior número de citações, seguido do Journal of Business Ethics. A quantidade de citações foi identificada através do indicador TCLS (Total Local Citation Score), indicador bibliométrico que mede o impacto de uma fonte por meio da quantidade de citações que essa recebeu no conjunto de trabalhos resultantes de uma busca (197 artigos). Se um periódico possui um indicador TCLS de 4 citações, por exemplo, significa que esse periódico foi citado por 4 dos 197 artigos analisados. Destaca-se que os dois periódicos com mais publicações também são os dois periódicos com mais citações.

\section{Resultados da análise sistemática dos artigos selecionados}

Nessa seção são apresentados os resultados das análises organizados em dois grupos. O primeiro se refere aos artigos mais citados e o segundo, aos artigos mais recentes.

\subsection{Grupo 1: artigos mais citados}

Considerando os trabalhos selecionados nesse grupo, os dois artigos mais citados são: Corporate Social Responsibility theories: Mapping the territory, de Garriga e Mele, publicado em 2004, citado 18 vezes, e Corporations, stakeholders and sustainable development I: A theoretical exploration of business-society relations, de Steurer et al., publicado em 2005, com 9 citações.

Os dois periódicos com maior número de citações e de artigos na área editaram 6 dos 13 artigos mais citados na pesquisa, evidenciando a forte concentração desses dois periódicos na temática.

Em relação à abordagem metodológica, são 8 estudos qualitativos e 5 quantitativos. Foram identificadas cinco problemáticas no grupo de artigos mais citados, 
Tabela 1. Periódicos com mais artigos publicados e/ou citados nas temáticas.

\begin{tabular}{|c|c|c|}
\hline Periódicos & $\begin{array}{c}\text { Quantidade de } \\
\text { publicações }\end{array}$ & $\begin{array}{c}\text { Quantidade de } \\
\text { citações }\end{array}$ \\
\hline Corporate Social Responsibility and Environmental Management & 43 & 57 \\
\hline Journal of Business Ethics & 39 & 45 \\
\hline Business Strategy and The Environment & 32 & 22 \\
\hline Management Decision & 7 & \\
\hline Business \& Society & 6 & 2 \\
\hline Organization \& Environment & 6 & 7 \\
\hline Supply Chain Management - An International Journal & 5 & 6 \\
\hline African Journal of Business Management & 3 & \\
\hline Journal of Organizational Change Management & 3 & \\
\hline Business Ethics - A European Review & 2 & \\
\hline European Journal of Marketing & 2 & 2 \\
\hline International Journal of Contemporary Hospitality Management & 2 & \\
\hline International Journal of Technology Management & 2 & \\
\hline Journal of International Management & 2 & \\
\hline Journal of Management & 2 & 14 \\
\hline Management International Review & 2 & \\
\hline Organization Studies & 2 & \\
\hline Total Quality Management \& Business Excellence & 2 & \\
\hline International Journal Of Consumer Studies & 2 & \\
\hline Public Relations Review & 2 & \\
\hline International Business Review & & 5 \\
\hline Scandinavian Journal of Management & & 4 \\
\hline Corporate Governance - An International Review & & 3 \\
\hline Entrepreneurship Theory And Practice & & 2 \\
\hline
\end{tabular}

Fonte: Elaboração dos autores.

sendo elas: Ontologia (3 artigos); Fatores para adoção de iniciativas de sustentabilidade (2 artigos); Avaliação de desempenho (3 artigos); Impactos sociais e/ou ambientais (3 artigos); e Relatórios de sustentabilidade ( 2 artigos). Tais problemáticas, que também se fizeram presentes na análise dos trabalhos mais recentes, são detalhadas na seção 5.2 a qual também apresenta as análises sistemáticas realizadas nos artigos mais recentes.

\subsection{Grupo 2: artigos mais recentes}

No grupo dos artigos mais recentes foram identificados 28 artigos publicados em 2015, 25 artigos publicados em 2014 e 22 artigos publicados em 2013. Dois periódicos concentram mais da metade das publicações na temática. O periódico Corporate Social Responsibility and Environmental Management publicou $31 \%$ dos artigos (23), sendo 8 publicados em 2013, 7 publicados em 2014 e 8 publicados em 2015. O outro periódico que se destaca na quantidade de publicações mais recentes é o Business Strategy and The Environment, com $29 \%$ das publicações mais recentes sobre o tema (22 artigos), sendo 4 artigos publicados em 2013, 8 em 2014 e 10 em 2015. Os demais 30 artigos selecionados foram publicados nos periódicos: Journal of Business
Ethics (10 artigos), Organization \& Environment (5 artigos), Business \& Society (2 artigos), Supply Chain Management - An International Journal (2 artigos), International Journal of Contemporary Hospitality Management (2 artigos), Management International Review (2 artigos), Management Decision (1 artigo), Business Ethics - A European Review (1 artigo), Organization Studies (1 artigo), Total Quality Management \& Business Excellence (1 artigo), International Journal of Consumer Studies (2 artigos) e Public Relations Review (1 artigo).

A abordagem metodológica aplicada aos artigos mais recentes segue a seguinte distribuição: 31 artigos qualitativos, 40 artigos quantitativos e 4 artigos quali-quantitativos. Nesse grupo foram identificadas sete problemáticas: Ontologia (3 artigos); Fatores para adoção de iniciativas de sustentabilidade (26 artigos); Avaliação de desempenho (16 artigos); Impactos sociais e/ou ambientais (15 artigos); Relatórios de sustentabilidade (9 artigos); Ensino (1 artigo); e Sustentabilidade como estratégia (5 artigos).

\section{Discussão}

A análise sistemática da literatura possibilitou a identificação de sete problemáticas nas quais concentram-se as publicações na temática. Dessas, 
cinco problemáticas foram encontradas tanto no grupo dos artigos mais citados quanto no grupo dos mais recentes: 1) Ontologia; 2) Fatores para adoção de iniciativas de sustentabilidade; 3) Avaliação de desempenho; 4) Impactos sociais e/ou ambientais; e 5) Relatórios de sustentabilidade. No grupo dos artigos mais recentes foram identificadas duas problemáticas adicionais: 6) Ensino; e 7) Sustentabilidade como estratégia. A partir da leitura na íntegra dos 88 artigos selecionados, analisaram-se os aspectos centrais que eram tratados principalmente nos objetivos e resultados. A partir dessa análise emergiram categorias de maneira indutiva, as quais foram tratadas por codificação aberta, resultando nas sete problemáticas apresentadas.

A problemática Ontologia contempla 6 artigos, o que representa aproximadamente $7 \%$ das obras analisadas neste trabalho. As obras dessa problemática visam definir o que é Responsabilidade Social Corporativa ou sustentabilidade empresarial (Kolk et al., 2014), trazendo os principais conceitos sobre as temáticas e também as convergências e divergências que os estudos têm contemplado (Garriga \& Mele, 2004; Lu \& Liu, 2014). Essa problemática caracteriza-se pela identificação dos elementos que definem RSC e Desenvolvimento Sustentável, apresentando alguns que são convergentes, como recursos ambientais e equidade social, e outros que expressam a visão mais particular do autor. Percebem-se definições amplas e sem um claro estabelecimento de fronteiras entre RSC e Desenvolvimento Sustentável, abrindo espaço para trabalhos que procurem delimitar melhor as interdependências entre as temáticas e uma taxonomia para o campo.

A problemática Fatores para adoção de iniciativas de sustentabilidade contempla os artigos que possuem como tema central apresentar fatores que motivam a adoção de iniciativas de sustentabilidade. Essa problemática contempla 28 artigos, o que corresponde a praticamente $32 \%$ dos estudos analisados. Dentre os fatores encontrados nos artigos, os stakeholders estão em destaque, por serem considerados reivindicadores às empresas (Steurer et al., 2005; Dashwood, 2014; Horisch et al., 2014) e responsáveis pela criação de conhecimento para o Desenvolvimento Sustentável (Siltaoja, 2014). Outros artigos tratam de quem são os diferentes stakeholders, como sociedade civil, ativistas, ONG, clientes e comunidade (Mzembe \& Meaton, 2014; Mahmood \& Humphrey, 2013; Ingenbleek \& Reinders, 2013; Burchell \& Cook, 2013) e governo. Outro fator em evidência é a liderança dos gestores, tendo eles potencial para internalizar a sustentabilidade nos processos de tomada de decisão organizacional (Nambiar \& Chitty, 2014), além de serem promovedores de bem-estar e importantes desenvolvedores da cultura organizacional (Alexander et al., 2014). Os gestores possuem influência significativa no desempenho ambiental nas empresas (Oberhofer \& Furst, 2013), sendo responsáveis pela difusão integrada de informações a respeito de sustentabilidade (Frias-Aceituno et al., 2013), sendo que a formação superior acadêmica dos gestores também mostrou-se um fator que influencia a adoção de iniciativas de sustentabilidade (Barrena-Martinez et al., 2015). O contexto de PME se mostra favorável ao desempenho financeiro, quando as dimensões econômica, social e ambiental da Responsabilidade Social Corporativa são associadas com as três capacidades específicas: visão compartilhada, gestão das partes interessadas e proatividade (Torugsa et al., 2013), sendo um ambiente participativo que permite a internalização de valores, comportamentos, crenças e ações necessárias para a adoção da Responsabilidade Social Empresarial (Stewart \& Gapp, 2014; Halme \& Korpela, 2014). Nessa problemática ficou nítida a relevância que, ao aprofundar as pressões e influências, a literatura dá aos stakeholders, com olhares mais particulares para fornecedores, clientes e comunidade, deixando uma lacuna para futuras pesquisas ao referir outros grupos de stakeholders. Destaca-se também o papel do líder e dos gestores na adoção de iniciativas sustentáveis. Outros fatores também foram identificados, mas poucas pesquisas buscaram investigar alguma relação entre eles. Aprofundar as relações entre os fatores já explorados, em direção a pesquisas quantitativas que busquem identificar fatores dependentes e independentes, por exemplo, configura-se mais um caminho para futuras pesquisas.

A Avaliação de desempenho é outra problemática identificada nesta pesquisa, a qual é tema de 19 artigos, o que representa aproximadamente $22 \%$ dos artigos analisados neste estudo. Essa problemática mostra que a busca de mensurar e perceber o desempenho da sustentabilidade empresarial se faz presente nas pesquisas. Alguns artigos apresentam que a relação de Responsabilidade Social Corporativa e resultado econômico pode ser negativa (Linder et al., 2014), ao menos quando esses indicadores de desempenho buscam mensurar resultados econômicos a curto prazo (Lopez et al., 2007). No entanto, a maioria dos estudos avalia o desempenho de responsabilidade social, competitividade e retorno financeiro como positivo (Callan \& Thomas, 2009; Wolf, 2013; Eisenbach et al., 2014; Hajmohammad \& Vachon, 2014; Delmas et al., 2015). No âmbito ambiental, entende-se que os esforços das empresas em matéria de proteção ambiental melhoram ainda mais seus valores de mercado (Linder et al., 2014). Também a inovação verde, que engloba melhorias tecnológicas que economizam energia, previnem a poluição ou permitem a reciclagem de resíduos está positivamente relacionada com rentabilidade (Aguilera-Caracuel \& Ortiz-de-Mandojana, 2013). O desenvolvimento e avaliação da sustentabilidade nas cadeias de fornecimento 
podem servir como ferramenta na identificação e avaliação de diversos indicadores de desempenho econômico, ambiental e social, auxiliando na tomada de decisões (Varsei et al., 2014). Mesmo em tempos de crise econômica, a sinergia entre o desempenho ambiental e financeiro é, ainda, forte. Sendo assim, as empresas devem continuar investindo em projetos sustentáveis, a fim de reforçar as relações com as suas partes interessadas e também elevar os seus lucros econômicos (Gallego-Álvarez et al., 2014), afinal, os consumidores são mais propensos a comprar produtos de empresas que adotam um posicionamento autobenéfico em relação à Responsabilidade Social Corporativa (Kim et al., 2014). Essa problemática está claramente delimitada pelo objeto de investigar a correlação entre desempenho financeiro e a implementação de iniciativas de sustentabilidade, evidenciando que os resultados podem ser diferentes considerando curto e longo prazo. Alguns artigos ressaltam que o desempenho pode ser visto não somente pela dimensão financeira. Sendo assim, futuras pesquisas poderiam propor um framework identificando as potenciais dimensões de desempenho relacionadas à sustentabilidade.

Os Impactos sociais e/ou ambientais são outra temática que os artigos que investigaram os impactos sociais e ambientais contemplam. Nessa problemática foram classificados 18 publicações, representando em torno de $20 \%$ dos artigos analisados. A Responsabilidade Social Corporativa contribui no desenvolvimento de resoluções de problemas de regiões mais carentes do mundo, como o desenvolvimento de instituições que contribuam para a justiça social, a proteção ambiental e a erradicação da pobreza (Dobers \& Halme, 2009), por meio de empresas que adotam políticas que abrangem questões de gênero, a representação formal dos trabalhadores, a distribuição de renda para as comunidades locais e a eliminação do trabalho infantil (Naeem \& Welford, 2009; Zhu et al., 2014). Alguns estudos focaram em grupos específicos como povos indígenas (Nikolakis et al., 2014) e comunidades sob impacto da pós-mineração (Littlewood, 2014). Empresas multinacionais são instituições que têm um papel específico, cujos impactos sociais e ambientais do negócio não são entendidos como problema mas sim parte da solução de problemas desse âmbito (Kolk \& Van Tulder, 2010). Os resultados da implantação de um sistema de gestão ambiental mostram o potencial da Responsabilidade Social Corporativa quando esse sistema é integrado na estratégia de negócios de empresas multinacionais, uma vez que entrega melhorias sociais e ambientais nos países em que as multinacionais estão instaladas (Garcia-Rodriguez et al., 2013). Interessante observar que as questões relativas a preservação do ambiente e equidade social são trabalhadas à luz do papel das organizações e de como essas impactam a sociedade.
Entender como a sociedade recebe esses impactos e responde a eles é uma outra lente que pode ser pesquisada, podendo auxiliar a orientar as práticas de RSC nas organizações.

Os Relatórios de sustentabilidade, outra problemática classificada neste trabalho, contempla 11 dos artigos analisados, sendo a sua representatividade em torno de $13 \%$. A aplicação de relatórios de sustentabilidade é motivada pelos fatores sociais e culturais da empresa, tendo uma influência sobre a intensidade da comunicação entre países. Entende-se que ainda podem efetivamente ser melhor elaborados (Fifka \& Drabble, 2012), necessitando de esforços agressivos para serem melhor estabelecidos (Amran et al., 2014). Ainda são pouco difundidas e aplicadas, por exemplo, as diretrizes da GRI, mas ressalta-se que a responsabilidade ambiental é muito importante para as empresas, inclusive mais do que a dimensão social (Steurer \& Konrad, 2009). Percebe-se que ajustes devem ser realizados nos relatórios de sustentabilidade, mas também se destaca a importância da sua utilização, visto que sua publicação pelas empresas fornece uma explicação mais ampla do desempenho empresarial, descrevendo a dependência e impactos da empresa em relação a diferentes recursos, seus relacionamentos (Frias-Aceituno et al., 2014), sendo ainda indicado que a empresa contrate um auditor para garantir as informações dos relatórios de sustentabilidade (Sierra et al., 2013). A comparação entre as empresas pode surgir por meio das divulgações de sustentabilidade pelos relatórios (Mio \& Venturelli, 2013). Ou seja, desenvolve-se um índice para medir a sustentabilidade, considerando que a elaboração de relatórios de Responsabilidade Social Corporativa impacta sobre a tomada de decisão das empresas (Zorio et al., 2013), sendo a aplicação de relatórios de sustentabilidade compreendida como a demonstração da conscientização das empresas sobre os impactos que seus produtos e processos podem causar ambiental e socialmente (Guziana \& Dobers, 2013). Essa problemática caracteriza-se por evidenciar a importância dos relatórios de sustentabilidade como mecanismos de comunicação, transparência, compliance e governança nas organizações. Dessa forma, investigar a relação entre o uso dos relatórios de sustentabilidade e avaliação de desempenho da organização (outra problemática identificada) pode ser uma questão para futuras pesquisas.

A problemática Ensino contou com apenas 1 artigo, o qual representa aproximadamente $1 \%$ dos artigos analisados. A problemática apresenta o quanto a responsabilidade social corporativa ainda deve ser explorada em maior intensidade, nos cursos e nas disciplinas das escolas de negócios, justamente por se considerar um tema relevante a ser aprofundado pela academia por meio do ensino (Doh \& Tashman, 2014). Explorar as temáticas como disciplinas a serem incorporadas aos currículos de ensino no sistema 
educacional, suas barreiras, consequências e desafios, mostra-se uma trilha promissora para pesquisas multidisciplinares entre Administração e Educação.

A Sustentabilidade como estratégia é mais uma problemática classificada nesta pesquisa, a qual é tema de 5 artigos, correspondendo a aproximadamente $5 \%$ dos estudos analisados. A sustentabilidade como estratégia pode ser orientada para a governança e gestão corporativa, englobando os âmbitos econômico, ecológico e social (Beckmann et al., 2014), sendo muito útil para análises internas e externas do negócio. Também é indicada a utilização da ISO 26000 para a melhor implementação da estratégia de sustentabilidade (Hahn, 2013). Constata-se também que há muitos desafios para a sustentabilidade ser compreendida como estratégia, mas também que as suas dimensões econômica, ambiental e social pertencem à medição do desempenho dos fornecedores, podendo as iniciativas de sustentabilidade ser uma estratégia empresarial (Morali \& Searcy, 2013). A sustentabilidade como estratégia é compreendida como um comportamento organizacional na adoção de estratégias ambientais proativas (Carballo-Penela \& Castromán-Diz, 2015). Nesse sentido, a proposição de modelos de maturidade para integração de sustentabilidade à estratégia da empresa, definindo características necessárias a cada nível, fica como uma sugestão para os pesquisadores da área.

\section{Considerações finais}

O presente estudo colabora para o entendimento das pesquisas que estão sendo desenvolvidas na área de Desenvolvimento Sustentável e Responsabilidade Social Corporativa através da exposição do cenário das pesquisas realizadas sobre essas temáticas, considerando suas características, contribuições e as problemáticas que abordam.
Os estudos provêm de diversos países: Holanda (Ingenbleek \& Reinders, 2013); Suécia (Linder et al., 2014); Itália e Reino Unido (Mio \& Venturelli, 2013); países asiáticos (Scholtens \& Kang, 2013), especificamente o Cazaquistão (Mahmood \& Humphrey, 2013) e países africanos (Mzembe \& Meaton, 2014). Todavia não se localizou nenhum estudo realizado no Brasil ou demais países da América do Sul, sendo essa uma oportunidade para pesquisas futuras.

A análise dos 88 artigos selecionados no grupo dos artigos mais citados (13 artigos) e artigos mais recentes (75 artigos) oferece um panorama das pesquisas em Desenvolvimento Sustentável e Responsabilidade Social Corporativa, apresentando uma taxonomia das problemáticas presentes na literatura corrente e identificando insights para futuras pesquisas (Figura 2).

Chama a atenção a quantidade de artigos na problemática Fatores para adoção de iniciativas de sustentabilidade. As problemáticas que surgiram no grupo dos artigos mais recentes - Ensino e Sustentabilidade como estratégia, podem apontar um caminho importante não só para futuras pesquisas mas também para o desenvolvimento da sociedade. Por um lado, já encontramos reforço em artigos aqui analisados na problemática Fatores para adoção de iniciativas de sustentabilidade, na qual a implantação da responsabilidade social corporativa está associada a especializações de ensino em administração e negócios que os gestores possuem (Huang, 2013), aumentando a possibilidade de uma visão mais estratégica de sustentabilidade. Por outro lado, promover a visão da temática Desenvolvimento Sustentável e RSC como disciplinas a serem incorporadas ao currículo de ensino no sistema educacional é um ponto muito importante para o aumento da consciência da sociedade em relação a questões que envolvem a continuidade dos recursos ambientais, bem como proporcionar
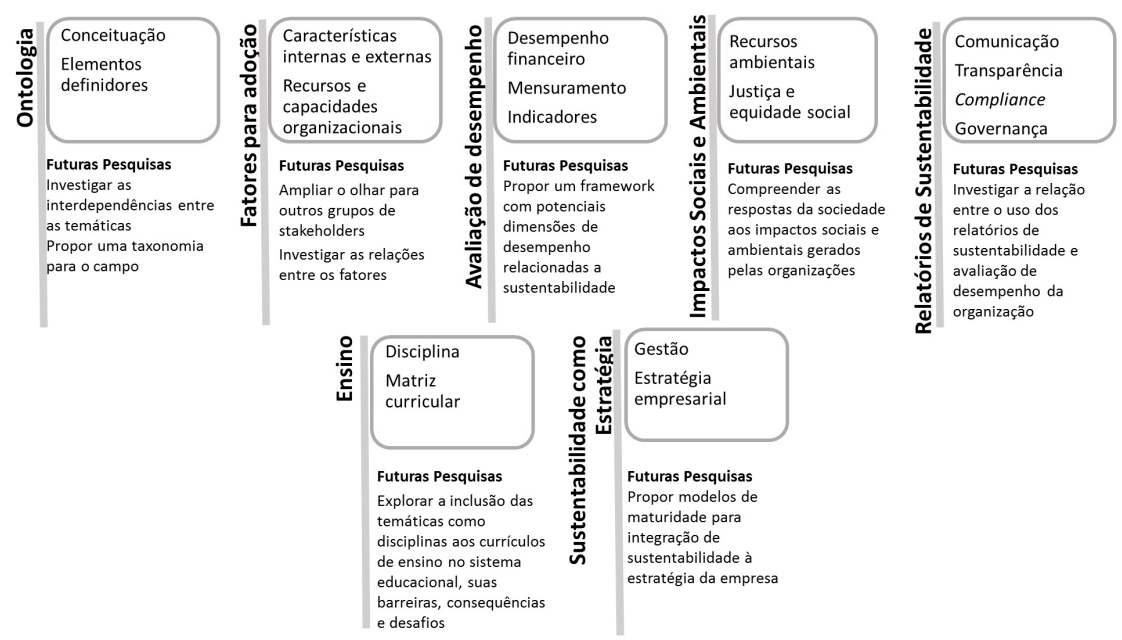

Figura 2. Problemáticas e insights para futuras pesquisas. Fonte: Elaboração dos autores. 
melhores condições sociais de forma mais igualitária. Da mesma forma, aprofundar pesquisas sobre sustentabilidade como estratégia empresarial pode levar as organizações a um nível maior de maturidade na sua relação com as práticas de Responsabilidade Social Corporativa, passando de comportamentos reativos e ações pontuais para uma maior integração de práticas socioambientais à estratégia de negócio. Além disso talvez seja possível uma consciência crescente em relação ao assunto, na qual atitudes de respeito ao próximo e ao ambiente passem a ser regra e não exceção. A influência das partes interessadas está presente em estudos de várias problemáticas, como: Fatores para adoção de iniciativas de sustentabilidade; Avaliação de desempenho; Impactos sociais e/ou ambientais; Relatórios de sustentabilidade; Ensino; e Sustentabilidade como estratégia, evidenciando a importância da teoria dos stakeholders como lente teórica nas pesquisas sobre o tema.

Por fim, percebe-se que pesquisas futuras podem ser desenvolvidas adotando-se diferentes abordagens metodológicas. Nas problemáticas Ontologia e Ensino, artigos conceituais e ensaios teóricos podem ser desenvolvidos buscando-se investigar as interdependências entre as temáticas e propor uma taxonomia para o campo ou explorar a inclusão das temáticas como disciplinas no currículo do sistema educacional, propondo-se um modelo de matriz curricular. Abordagens qualitativas podem atender a problemática Impactos sociais e/ou ambientais, ampliando o olhar sobre tais impactos. As pesquisas atuais os estudam à luz do papel das organizações e de como essas impactam a sociedade. Entender como a sociedade recebe esses impactos e responde a eles é um outro olhar a ser pesquisado. As problemáticas Fatores para adoção de iniciativas de sustentabilidade e Relatórios de sustentabilidade podem adotar abordagens quantitativas testando hipóteses que correlacionem ou identifiquem relações de dependência entre os fatores, para adoção de iniciativas de sustentabilidade, ou entre o uso de relatórios de sustentabilidade e variáveis de desempenho organizacional. As problemáticas Avaliação de desempenho e Sustentabilidade como estratégia poderiam adotar abordagens mixmetodos (Quali-quanti) para propor frameworks com potenciais dimensões de desempenho relacionadas a sustentabilidade ou modelos de maturidade para integração de sustentabilidade à estratégia da empresa.

\section{Referências}

Aguilera-Caracuel, J., \& Ortiz-de-Mandojana, N. (2013). Green innovation and financial performance: an institutional approach. Organization \& Environment, 26(4), 365-385. http://dx.doi.org/10.1177/1086026613507931.

Alexander, A., Walker, H., \& Naim, M. (2014). Decision theory in sustainable supply chain management: a literature review. Supply Chain Management-An International Journal, 19(5-6), 504-522. http://dx.doi.org/10.1108/ SCM-01-2014-0007.

Amran, A., Lee, S. P., \& Devi, S. S. (2014). The influence of governance structure and strategic corporate social responsibility toward sustainability reporting quality. Business Strategy and the Environment, 23(4), 217-235. http://dx.doi.org/10.1002/bse.1767.

Barrena-Martinez, J., Lopez-Fernandez, M., MarquezMoreno, C., \& Romero-Fernandez, P. M. (2015). Corporate social responsibility in the process of attracting college graduates. Corporate Social Responsibility and Environmental Management, 22(6), 408-423. http:// dx.doi.org/10.1002/csr.1355.

Barros, R. F. (2007). Desenvolvimento regional sustentável: a experiência do Banco do Brasil (Dissertação de mestrado). Programa de Pós-graduação em Administração, Universidade de Brasília, Brasília.

Beckmann, M., Hielscher, S., \& Pies, I. (2014). Commitment strategies for sustainability: how business firms can transform trade-offs into win-win outcomes. Business Strategy and the Environment, 23(1), 18-37. http:// dx.doi.org/10.1002/bse.1758.

Bignetti, L. P., \& Paiva, E. L. (2002). Ora (direis) ouvir estrelas! Estudo das citações de autores de estratégia na produção acadêmica brasileira. Revista de Administração Contemporânea, 6(1), 105-125. http://dx.doi.org/10.1590/ S1415-65552002000100007.

Brambilla, S. D., \& Stumpf, I. R. C. (2012). Produção científica da UFRGS representada na WOS (2000-2009). Perspectivas em Ciência da Informação, 17(3), 34-50. http://dx.doi.org/10.1590/S1413-99362012000300004.

Brundtland, G. H. (1987). Our common future: the World Commission on Environment and Development. Oxford: Oxford University Press.

Burchell, J., \& Cook, J. (2013). CSR, Co-optation and resistance: the emergence of new agonistic relations between business and civil society. Journal of Business Ethics, 115(4), 741-754. http://dx.doi.org/10.1007/ s10551-013-1830-z.

Callan, S. J., \& Thomas, J. M. (2009). Corporate financial performance and corporate social performance: an update and reinvestigation. Corporate Social Responsibility and Environmental Management, 16(2), 61-78. http:// dx.doi.org/10.1002/csr.182.

Carballo-Penela, A., \& Castromán-Diz, J. L. (2015). Environmental policies for sustainable development: an analysis of the drivers of proactive environmental strategies in the service sector. Business Strategy and the Environment, 24(8), 802-818. http://dx.doi. org/10.1002/bse.1847.

Carroll, A. B. (1979). A three dimensional model of corporate performance. Academy of Management Review, 4, 497-505. 
Crossan, M., \& Apaydin, M. (2010). A multi-dimensional framework of organizational innovation: a systematic review of the literature. Journal of Management Studies, 47(6), 1154-1191. http://dx.doi.org/10.1111/j.14676486.2009.00880.x.

Dashwood, H. S. (2014). Sustainable development and industry self-regulation: developments in the global mining sector. Business \& Society, 53(4), 551-582. http://dx.doi.org/10.1177/0007650313475997.

Delmas, M. A., Nairn-Birch, N., \& Lim, J. (2015). Dynamics of environmental and financial performance the case of greenhouse gas emissions. Organization \& Environment, 28(4), 374-393. http://dx.doi. org/10.1177/1086026615620238.

Desjardins, J. (1998). Corporate environmental responsibility. Journal of Business Ethics, 17(8), 825-838. http://dx.doi. org/10.1023/A:1005719707880.

Dobers, P., \& Halme, M. (2009). Corporate social responsibility and developing countries. Corporate Social Responsibility and Environmental Management, 16(5), 237-249. http://dx.doi.org/10.1002/csr.212.

Doh, J. P., \& Tashman, P. (2014). Half a world away: the integration and assimilation of corporate social responsibility, sustainability, and sustainable development in business school curricula. Corporate Social Responsibility and Environmental Management, 21(3), 131-142. http:// dx.doi.org/10.1002/csr.1315.

Eisenbach, S., Schiereck, D., Trillig, J., \& Von Flotow, P. (2014). Sustainable project finance, the adoption of the equator principles and shareholder value effects. Business Strategy and the Environment, 23(6), 375-394. http://dx.doi.org/10.1002/bse.1789.

Elkington, J. (1997). Cannibals with forks: the triple bottom line of 21 st century business. Gabriola Island: New Society Publishers.

Fifka, M. S., \& Drabble, M. (2012). Focus and standardization of sustainability reporting: a comparative study of the United Kingdom and Finland. Business Strategy and the Environment, 21(7), 455-474. http://dx.doi. org/10.1002/bse. 1730 .

Frias-Aceituno, J. V., Rodriguez-Ariza, L., \& Garcia-Sanchez, I. M. (2013). The role of the board in the dissemination of integrated corporate social reporting. Corporate Social Responsibility and Environmental Management, 20(4), 219-233. http://dx.doi.org/10.1002/csr.1294.

Frias-Aceituno, J. V., Rodriguez-Ariza, L., \& GarciaSanchez, I. M. (2014). Explanatory factors of integrated sustainability and financial reporting. Business Strategy and the Environment, 23(1), 56-72. http://dx.doi. org/10.1002/bse. 1765 .

Gallego-Álvarez, I., García-Sánchez, I. M., \& Vieira, C. D. (2014). Climate change and financial performance in times of crisis. Business Strategy and the Environment, 23(6), 361-374. http://dx.doi.org/10.1002/bse.1786.
Garcia-Rodriguez, F. J., Garcia-Rodriguez, J. L., CastillaGutierrez, C., \& Major, S. A. (2013). Corporate Social Responsibility of oil companies in developing countries: from altruism to business strategy. Corporate Social Responsibility and Environmental Management, 20(6), 371-384. http://dx.doi.org/10.1002/csr.1320.

Garriga, E., \& Mele, D. N. (2004). Corporate Social Responsibility theories: mapping the territory. Journal of Business Ethics, 53(1-2), 51-71. http://dx.doi. org/10.1023/B:BUSI.0000039399.90587.34.

Goulart, S., \& Carvalho, C. A. (2008). O caráter da internacionalização da produção científica e sua acessibilidade restrita. Revista de Administração Contemporânea, 12(3), 835-853. http://dx.doi.org/10.1590/ S1415-65552008000300011.

Guziana, B., \& Dobers, P. (2013). How sustainability leaders communicate corporate activities of sustainable development. Corporate Social Responsibility and Environmental Management, 20(4), 193-204. http:// dx.doi.org/10.1002/csr.1292.

Hahn, R. (2013). ISO 26000 and the Standardization of Strategic Management Processes for Sustainability and Corporate Social Responsibility. Business Strategy and the Environment, 22(7), 442-455. http://dx.doi. org/10.1002/bse. 1751 .

Hajmohammad, S., \& Vachon, S. (2014). Safety culture: a catalyst for sustainable development. Journal of Business Ethics, 123(2), 263-281. http://dx.doi.org/10.1007/ s10551-013-1813-0.

Halme, M., \& Korpela, M. (2014). Responsible innovation toward sustainable development in small and mediumsized enterprises: a resource perspective. Business Strategy and the Environment, 23(8), 547-566. http:// dx.doi.org/10.1002/bse.1801.

Horisch, J., Freeman, R. E., \& Schaltegger, S. (2014). Applying stakeholder theory in sustainability management: links, similarities, dissimilarities, and a conceptual framework. Organization \& Environment, 27(4), 328-346. http:// dx.doi.org/10.1177/1086026614535786.

Huang, S. K. (2013). The Impact of CEO Characteristics on Corporate Sustainable Development. Corporate Social Responsibility and Environmental Management, 20(4), 234-244. http://dx.doi.org/10.1002/csr.1295.

Ingenbleek, P. T. M., \& Reinders, M. J. (2013). The development of a market for sustainable coffee in the netherlands: rethinking the contribution of fair trade. Journal of Business Ethics, 113(3), 461-474. http:// dx.doi.org/10.1007/s10551-012-1316-4.

Iritani, D. R., Morioka, S. N., Carvalho, M. M. D., \& Ometto, A. R. (2015). Análise sobre os conceitos e práticas de Gestão por Processos: revisão sistemática e bibliometria. Gestão \& Produção, 22(1), 164-180. http://dx.doi.org/10.1590/0104-530X814-13.

Kim, M. S., Kim, D. T., \& Kim, J. I. (2014). CSR for Sustainable Development: CSR beneficiary positioning 
and impression management motivation. Corporate Social Responsibility and Environmental Management, 21(1), 14-27. http://dx.doi.org/10.1002/csr.1300.

Kolk, A., \& Van Tulder, R. (2010). International business, corporate social responsibility and sustainable development. International Business Review, 19(2), 119-125. http:// dx.doi.org/10.1016/j.ibusrev.2009.12.003.

Kolk, A., Rivera-Santos, M., \& Rufin, C. (2014). Reviewing a decade of research on the Base/Bottom of the Pyramid (BOP) Concept. Business \& Society, 53(3), 338-377. http://dx.doi.org/10.1177/0007650312474928.

Kurtz, D. J., Santos, J. L. S., \& Steil, A. V. (2013). Capacidade de absorção do conhecimento e capacidades dinâmicas no contexto de ambientes turbulentos: uma análise da literatura. In Anais do IV Encontro de Administração da Informação (EnADI). Bento Gonçalves: ANPAD.

Laruccia, M. M. (2012). Sustainability strategies: when does it pay to be green? Revista de Administração Contemporânea, 16(1), 172-174. http://dx.doi.org/10.1590/ S1415-65552012000100011.

Lazzarotti, F., Dalfovo, M. S., \& Hoffmann, V. E. (2011). A bibliometric study of innovation based on Schumpeter. Journal of Technology Management \& Innovation. Santiago, 6(4), 121-135. http://dx.doi.org/10.4067/ S0718-27242011000400010.

Linder, M., Bjorkdahl, J., \& Ljungberg, D. (2014). Environmental orientation and economic performance: a quasi-experimental study of small swedish firms. Business Strategy and the Environment, 23(5), 333-348. http://dx.doi.org/10.1002/bse.1788.

Littlewood, D. (2014). Cursed' communities? Corporate Social Responsibility (CSR), company towns and the mining industry in Namibia. Journal of Business Ethics, 120(1), 39-63. http://dx.doi.org/10.1007/s10551-0131649-7.

Lopez, M. V., Garcia, A., \& Rodriguez, L. (2007). Sustainable development and corporate performance: a study based on the Dow Jones Sustainability Index. Journal of Business Ethics, 75(3), 285-300. http://dx.doi. org/10.1007/s10551-006-9253-8.

Lu, L. Y. Y., \& Liu, J. S. (2014). The knowledge diffusion paths of corporate social responsibility: from 1970 to 2011. Corporate Social Responsibility and Environmental Management, 21(2), 113-128. http://dx.doi.org/10.1002/ csr.1309.

Lyra, M. G., Gomes, R. C., \& Jacovine, L. A. G. (2009). O papel dos stakeholders na sustentabilidade da empresa: contribuições para construção de um modelo de análise. Revista de Administração Contemporânea, 13(spe), 39-52. http://dx.doi.org/10.1590/S1415-65552009000500004.

Machado, R. N. (2007). Análise cientométrica dos estudos bibliométricos publicados em periódicos da área de biblioteconomia e ciência da informação (1990-2005). Perspectiva em Ciência da Informação, 12(3), 2-20. http://dx.doi.org/10.1590/S1413-99362007000300002.
Mahmood, M., \& Humphrey, J. (2013). Stakeholder expectation of corporate social responsibility practices: a study on local and multinational corporations in Kazakhstan. Corporate Social Responsibility and Environmental Management, 20(3), 168-181. http:// dx.doi.org/10.1002/csr. 1283 .

Melo, P. L. R., \& Andreassi, T. (2010). Publicação científica nacional e internacional sobre franchising: levantamento e análise do período 1998-2007. Revista de Administração Contemporânea, 14(2), 268-288. http://dx.doi.org/10.1590/S1415-65552010000200006.

Mio, C., \& Venturelli, A. (2013). Non-financial information about sustainable development and environmental policy in the annual reports of listed companies: evidence from italy and the UK. Corporate Social Responsibility and Environmental Management, 20(6), 340-358. http:// dx.doi.org/10.1002/csr.1296.

Montiel, I., \& Delgado-Ceballos, J. (2014). Defining and measuring corporate sustainability: are we there yet? Organization \& Environment, 27(2), 113-139. http:// dx.doi.org/10.1177/1086026614526413.

Morali, O., \& Searcy, C. (2013). A review of sustainable supply chain management practices in Canada. Journal of Business Ethics, 117(3), 635-658. http://dx.doi. org/10.1007/s10551-012-1539-4.

Moretti, S. L. A., \& Campanário, M. A. (2009). A produção intelectual brasileira em Responsabilidade Social Empresarial - RSE sob a ótica da Bibliometria. $R A C$, $13,68-86$.

Mzembe, A. N., \& Meaton, J. (2014). Driving corporate social responsibility in the malawian mining industry: a stakeholder perspective. Corporate Social Responsibility and Environmental Management, 21(4), 189-201. http:// dx.doi.org/10.1002/csr.1319.

Naeem, M. A., \& Welford, R. (2009). A comparative study of corporate social responsibility in Bangladesh and Pakistan. Corporate Social Responsibility and Environmental Management, 16(2), 108-122. http:// dx.doi.org/10.1002/csr.185.

Nambiar, P., \& Chitty, N. (2014). Meaning making by managers: corporate discourse on environment and sustainability in India. Journal of Business Ethics, 123(3), 493-511. http://dx.doi.org/10.1007/s10551-013-1848-2.

Nikolakis, W., Nelson, H. W., \& Cohen, D. H. (2014). Who pays attention to indigenous peoples in sustainable development and why? Evidence from socially responsible investment mutual funds in North America. Organization \& Environment, 27(4), 368-382. http:// dx.doi.org/10.1177/1086026614546812.

Oberhofer, P., \& Furst, E. (2013). Sustainable development in the transport sector: influencing environmental behaviour and performance. Business Strategy and the Environment, 22(6), 374-389. http://dx.doi.org/10.1002/ bse. 1750 . 
Payne, D. M., \& Raiborn, C. A. (2001). Sustainable development: the ethics support the economics. Journal of Business Ethics, 32(2), 157-168. http://dx.doi. org/10.1023/A:1010726830191.

Santos, J. L. S., Uriona-Maldonado, M., \& Santos, R. N. M. (2011). Inovação e Conhecimento organizacional: um mapeamento bibliométrico das publicações científicas até 2009. Organizações em Contexto, 7(13), 31-58. http://dx.doi.org/10.15603/1982-8756/roc.v7n13p31-58.

Scholtens, B., \& Kang, F. C. (2013). Corporate social responsibility and earnings management: evidence from asian economies. Corporate Social Responsibility and Environmental Management, 20(2), 95-112. http:// dx.doi.org/10.1002/csr.1286.

Sierra, L., Zorio, A., \& Garcia-Benau, M. A. (2013). Sustainable development and assurance of corporate social responsibility reports published by ibex-35 companies. Corporate Social Responsibility and Environmental Management, 20(6), 359-370. http:// dx.doi.org/10.1002/csr.1303.

Siltaoja, M. E. (2014). Revising the corporate social performance model: towards knowledge creation for sustainable development. Business Strategy and the Environment, 23(5), 289-302. http://dx.doi.org/10.1002/ bse. 1783 .

Singleton, R. A., \& Straits, B. C. (1999). Approaches to social research. New York: Oxford University Press.

Souza, M. T. S., \& Ribeiro, H. C. M. (2013). Sustentabilidade ambiental: uma meta-análise da produção brasileira em periódicos de administração. Revista de Administração Contemporânea, 17(3), 368-396. http://dx.doi.org/10.1590/ S1415-65552013000300007.

Steurer, R., \& Konrad, A. (2009). Business-society relations in Central-Eastern and Western Europe: How those who lead in sustainability reporting bridge the gap in corporate (social) responsibility. Scandinavian Journal of Management, 25(1), 23-36. http://dx.doi.org/10.1016/j. scaman.2008.11.001.

Steurer, R., Langer, M. E., Konrad, A., \& Martinuzzi, A. (2005). Corporations, stakeholders and sustainable development I: a theoretical exploration of businesssociety relations. Journal of Business Ethics, 61(3), 263-281. http://dx.doi.org/10.1007/s10551-005-7054-0.

Stewart, H., \& Gapp, R. (2014). Achieving effective sustainable management: a small-medium enterprise case study. Corporate Social Responsibility and Environmental Management, 21(1), 52-64. http://dx.doi. org/10.1002/csr.1305.

Torugsa, N. A., O'donohue, W., \& Hecker, R. (2013). Proactive CSR: an empirical analysis of the role of its economic, social and environmental dimensions on the association between capabilities and performance. Journal of Business Ethics, 115(2), 383-402. http:// dx.doi.org/10.1007/s10551-012-1405-4.

Tranfield, D. R., Denyer, D., \& Smart, P. (2003). Towards a methodology for developing evidence-informed management knowledge by means of systematic review. British Journal of Management, 14(3), 207-222. http:// dx.doi.org/10.1111/1467-8551.00375.

Varsei, M., Soosay, C., Fahimnia, B., \& Sarkis, J. (2014). Framing sustainability performance of supply chains with multidimensional indicators. Supply Chain ManagementAn International Journal, 19(3), 242-257. http://dx.doi. org/10.1108/SCM-12-2013-0436.

Watanuki, H. M., Nadae, J., Carvalho, M. M., \& Moraes, R. O. (2014). Gestão de projetos internacionais: um estudo bibliométrico. Gestão \& Produção, 21(3), 660675. http://dx.doi.org/10.1590/0104-530X394.

Wolf, J. (2013). Improving the sustainable development of firms: the role of employees. Business Strategy and the Environment, 22(2), 92-108. http://dx.doi.org/10.1002/ bse. 1731 .

Yarime, M., Takeda, Y., \& Kajikawa, Y. (2010). Towards institutional analysis of sustainability science: a quantitative examination of the patterns of research collaboration. Sustainability Science, 5(1), 115-125. http://dx.doi.org/10.1007/s11625-009-0090-4.

Zhu, Q., Yin, H., Liu, J., \& Lai, K. (2014). How is employee perception of organizational efforts in corporate social responsibility related to their satisfaction and loyalty towards developing harmonious society in chinese enterprises? Corporate Social Responsibility and Environmental Management, 21(1), 28-40. http://dx.doi. org/10.1002/csr.1302.

Zorio, A., Garcia-Benau, M. A., \& Sierra, L. (2013). Sustainability development and the quality of assurance reports: empirical evidence. Business Strategy and the Environment, 22(7), 484-500. http://dx.doi.org/10.1002/ bse. 1764 . 